Article

\title{
Synthesis and Biological Activity of Benzamides Substituted with Pyridine-Linked 1,2,4-Oxadiazole
}

\author{
Sen Yang, Xiao-Yu Tian, Tian-Yang Ma, Li Dai, Chao-Li Ren, Jun-Chang Mei, Xing-Hai Liu and \\ Cheng-Xia Tan * \\ College of Chemical Engineering, Zhejiang University of Technology, Hangzhou 310014, China; \\ y17764528124@163.com (S.Y.); t895745887@163.com (X.-Y.T.); m15755187338@163.com (T.-Y.M.); \\ 15157357729@163.com (L.D.); 18708589843@163.com (C.-L.R.); meijunchang_ucb@163.com (J.-C.M.); \\ xhliu@zjut.edu.cn (X.-H.L.) \\ * Correspondence: tanchengxia@zjut.edu.cn; Tel./Fax: +86-571-8832-0238
}

Received: 15 July 2020; Accepted: 31 July 2020; Published: 31 July 2020

\begin{abstract}
To find pesticidal lead compounds with high activity, a series of novel benzamides substituted with pyridine-linked 1,2,4-oxadiazole were designed by bioisosterism, and synthesized easily via esterification, cyanation, cyclization and aminolysis reactions. The structures of the target compounds were confirmed by ${ }^{1} \mathrm{H}-\mathrm{NMR},{ }^{13} \mathrm{C}-\mathrm{NMR}$ and HRMS. The preliminary bioassay showed that most compounds had good larvicidal activities against mosquito larvae at $10 \mathrm{mg} / \mathrm{L}$, especially compound 7a, with a larvicidal activity as high as $100 \%$, and even at $1 \mathrm{mg} / \mathrm{L}$ was still $40 \%$; at $50 \mathrm{mg} / \mathrm{L}$, all the target compounds showed good fungicidal activities against the eight tested fungi. Moreover, compound $7 \mathrm{~h}$ exhibited better inhibitory activity (90.5\%) than fluxapyroxad (63.6\%) against Botrytis cinereal. Therefore, this type of compound can be further studied.
\end{abstract}

Keywords: amide compound; fungicidal activity; insecticidal activity; 1,2,4-oxadiazole; synthesis

\section{Introduction}

Recently, the demand for pesticides has been increasing with the improvement of people's living standards. Many new kinds of pesticides with high efficiency, low toxicity and low residue have been developed. Heterocyclic derivatives received important attention due to their various biological activities [1-6]. Among these compounds, 1,2,4-oxadiazole heterocyclic derivatives, which contain nitrogen and oxygen atoms, displayed diverse activities, such as insecticidal activity [7-9], antifungal activity [10,11], herbicidal activity [12], anti-inflammatory [13], hypotensive [14] and other physiological activity. In addition, amide groups could easily generate hydrogen bonds with the activated part of the target enzymes and control the target organisms. Moreover, introducing the amide structure was beneficial for the biodegradation of pesticides. So, amide derivatives were wildly studied [15-20], such as the commercial herbicide flufenacet (Figure 1), insecticide flubendiamide (Figure 1) and fungicide flutolanil (Figure 1).

Amide compounds could be roughly classified as carboxyamides, mandelic acid amides and phenylamides according to their chemical structures. Through analyzing the reported amide compounds, it was found that when the carboxylic acid parts of the compounds include the structural units of pyridine-linked heterocycle, amide compounds usually have better insecticidal or fungicidal activities. Most of the compounds that have been reported include the structural units of pyridine-linked pyrazole, such as the commercial insecticide chlorantraniliprole (Figure 1) and the compound A (Figure 1, at $50 \mathrm{mg} / \mathrm{L}$, its fungicidal activity against Botrytis cinerea was $80 \%$ ) synthesized by $\mathrm{Xu}$ et al. [21]. However, there are few reports about pyridine-linked 1,2,4-oxadiazole. Moreover, Syngenta has reported a lot of 1,2,4-oxadiazole substituted benzamides such as compounds B (Figure 1) 
and $\mathbf{C}$ (Figure 1) that had good fungicidal activities [22,23]. In view of these facts mentioned above, to find pesticides with high biological activity, using $\mathbf{A}$ and $\mathbf{B}$ as lead compounds and replacing the structure of trifluoromethyl with the pyridine ring, a series of novel benzamides substituted with pyridine-linked 1,2,4-oxadiazole have been designed and synthesized according to bioisosterism (Figure 2). Target compounds were confirmed by ${ }^{1} \mathrm{H}-\mathrm{NMR},{ }^{13} \mathrm{C}-\mathrm{NMR}$ and HRMS. Their insecticidal and fungicidal activities were studied and the result showed that the target compounds had good insecticidal and fungicidal activities. The synthetic route of the target compounds is shown in Scheme 1.

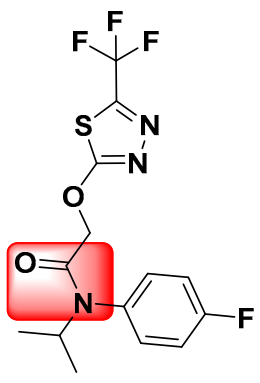

flufenacet

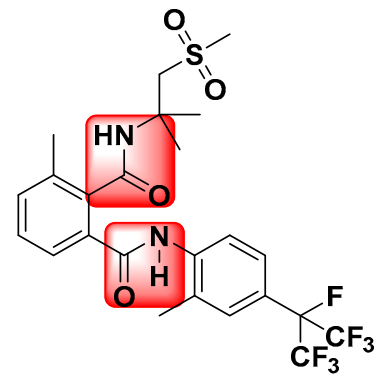

flubendiamide

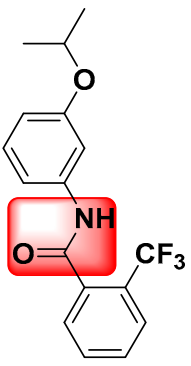

flutolanil

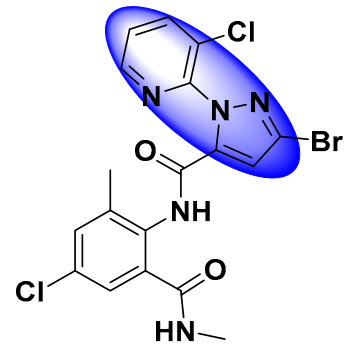

chlorantraniliprole

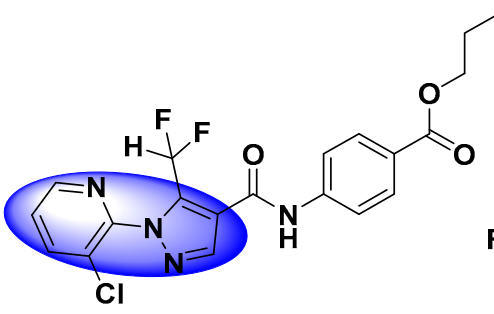

A

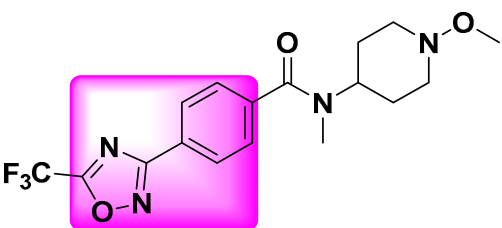

B

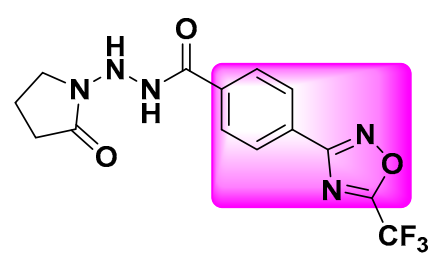

C

Figure 1. Chemical structures of flufenacet, flubendiamide, flutolanil, chlorantraniliprole and compounds $\mathbf{A}, \mathbf{B}, \mathbf{C}$.

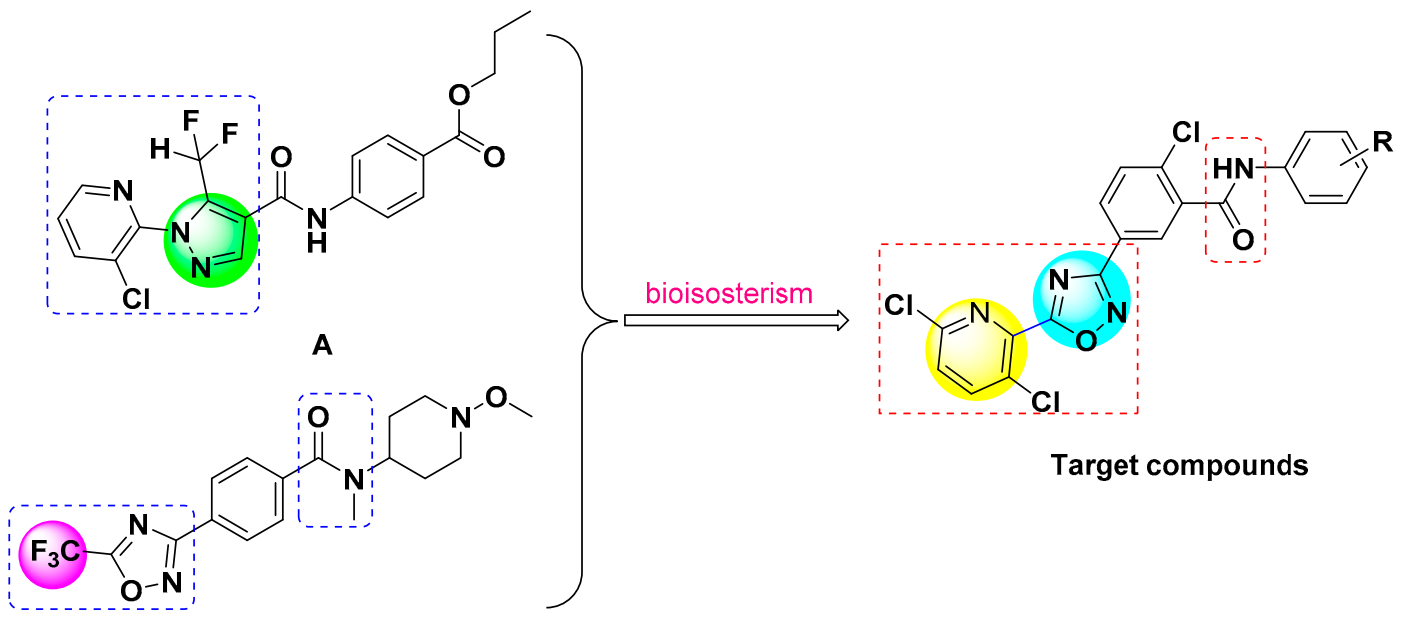

B

Figure 2. Design strategy of the target compounds. 
<smiles>COC(=O)c1cc(C#N)ccc1Cl</smiles><smiles>[R]c1ccc(NC(=O)c2cc(-c3noc(-c4nc(Cl)ccc4Cl)n3)ccc2C(C)C)c(Cl)c1</smiles>

Scheme 1. Synthetic route of target compound 7.

\section{Results and Discussion}

\subsection{Synthesis of Target Compounds}

The starting material 2-chloro-5-iodobenzoic acid 1 experienced esterification and cyanation reaction to give methyl 2-chloro-5-cyanobenzoate 3. Methyl 2-chloro-5-cyanobenzoate 3 then reacted with $\mathrm{NH}_{2} \mathrm{OH} \bullet \mathrm{HCl}$ under the alkaline condition to give amine oxime. This reaction could not be carried out for a long time, because amide byproducts would have formed easily. After this step, 2-chloro-5-(5-(3,6-dichloropyridin-2-yl)-1,2,4-oxadiazol-3-yl) benzoate 5 was synthesized by cyclization reaction from compounds 4 with 3,6-dichloropicolinoyl chloride that had been synthesized from 3,6-dichloropicolinic acid. At last, the intermediate 5 went through hydrolysis reaction, and then reacted with substituted aniline to give the target compound 7 .

In step 2, the iodine atom on the benzene ring being replaced by a cyano group is a typical nucleophilic substitution reaction. $\mathrm{NaCN}$ and $\mathrm{KCN}$ are two common nucleophilic reagents. In fact, these two reagents are highly toxic, so we chose $\mathrm{CuCN}$, which has relatively low toxicity, as the cyanidation reagent to reduce the risk in the experiment and the harm to the environment. In addition, the process of our experiment was different from that reported in former papers [24,25]. Using small natural organic molecule L-proline as the catalyst, DMF as the solvent and under the condition where temperature gradually increased, the amount of by-product decreased and the yield of product $\mathbf{3}$ was the highest [26]. The influence of different experimental conditions on the yield of product 3 is shown in Table 1.

Table 1. Effects of reaction conditions on the synthesis of the intermediate 3 .

\begin{tabular}{cccc}
\hline Entry & Catalyst & Condition & Yield/\% \\
\hline 1 & no & $100^{\circ} \mathrm{C}$ for $11 \mathrm{~h}$ & hardly react \\
2 & L-proline & $100{ }^{\circ} \mathrm{C}$ for $11 \mathrm{~h}$ & 66 \\
3 & L-proline & $70^{\circ} \mathrm{C}$ for $2 \mathrm{~h}$, further $100^{\circ} \mathrm{C}$ for $9 \mathrm{~h}$ & 79 \\
4 & L-proline & $70^{\circ} \mathrm{C}$ for $3 \mathrm{~h}$, further $100^{\circ} \mathrm{C}$ for $7 \mathrm{~h}$ & 73 \\
5 & L-proline & $80^{\circ} \mathrm{C}$ for $2 \mathrm{~h}$, further $100^{\circ} \mathrm{C}$ for $9 \mathrm{~h}$ & 75 \\
\hline
\end{tabular}


In step 4, the formation of 1,2,4-oxadiazole was achieved in a one-pot reaction. 3,6-dichloropicolinoyl chloride that was freshly prepared was dropped into the solution of methyl-2-chloro-5-( $N^{\prime}$-hydroxycarbamimidoyl) benzoate 4 and triethylamine in toluene at $0{ }^{\circ} \mathrm{C}$, to give the intermediate of methyl-2-chloro-5-(N-(3,6-dichloropicolinoyl)- $N^{\prime}$-hydroxycarbamimidoyl)benzoate. Next, the intermediate was cyclized to produce 1,2,4-oxadiazole at reflux (Scheme 2). In this way, the self-cyclization of compound 4 was avoided because of the higher reactivity of acid chloride.

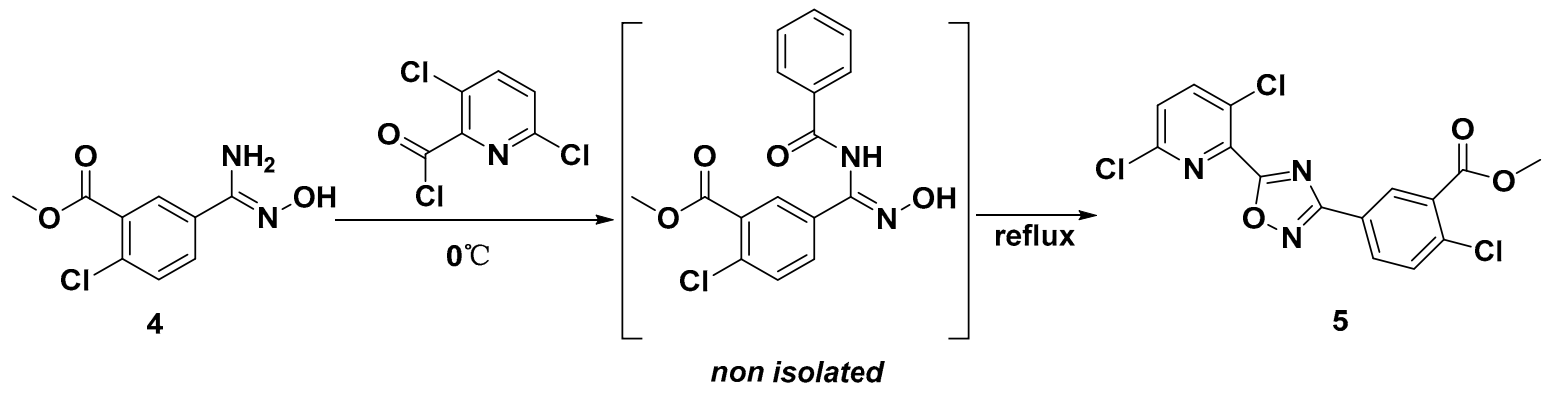

Scheme 2. Reaction equation of step 4 .

\subsection{Spectrum Analysis of Target Compounds}

The target compound 7a was taken as an example to conduct spectrum analysis. In the ${ }^{1} \mathrm{H}-\mathrm{NMR}$ spectra of compound 7a, the singlet at $\delta 10.09$ ppm was the NH peak. The signals of benzene and pyridine rings were assigned at $8.37-7.04 \mathrm{ppm}$. In the ${ }^{13} \mathrm{C}-\mathrm{NMR}$ spectra of compound $7 \mathbf{a}$, the $\mathrm{C}=\mathrm{O}$ signal could be found at $171.75 \mathrm{ppm}$. The appearance of signals at 167.27 and $163.91 \mathrm{ppm}$ was assigned to the carbons of the 1,2,4-oxadiazole ring. In the HRMS spectrogram, the calculated value of the ion peak of this compound was $[\mathrm{M}+\mathrm{Na}]^{+} 466.9840$, and the measured value was $[\mathrm{M}+\mathrm{Na}]^{+} 466.9840$. The absolute error was within 0.003 .

\subsection{Biological Activities of Target Compounds}

The results of the insecticidal activity test of the target compounds are shown in Tables 2 and 3. In Table 2, the death rates of compound 7 are all below 50\% against mythimna sepatara, helicoverpa armigera and pyrausta nubilalis at $500 \mathrm{mg} / \mathrm{L}$. Therefore, the insecticidal activities of this series of compounds were not good enough against the three targets. Nevertheless, we can see that compound 7 exhibited good larvicidal activity against mosquito larvae from Table 3 . The larvicidal activities of compounds $\mathbf{7 a}$ and $\mathbf{7 f}$ were $100 \%$ at $10 \mathrm{mg} / \mathrm{L}$. Furthermore, it was found that the larvicidal activity of compound 7 a was $100 \%$ at $2 \mathrm{mg} / \mathrm{L}$. Even at $1 \mathrm{mg} / \mathrm{L}$, the larvicidal activity was still $40 \%$. It exhibited better larvicidal potency than etoxazole against mosquito larvae. It can be seen from the compound $7 \mathbf{h}, 7 \mathbf{i}$ and $7 \mathbf{j}$ that the position of the substituent has little effect on the larvicidal activity of the target compound. Furthermore, as for compounds $\mathbf{7 a}$ to $\mathbf{7 n}$, from the general trend in the larvicidal activity, it can be concluded that the less steric substitution attached to aniline may reduce the obstacles of the target compound binding to the target receptor and help to bring about an increase in activity.

The results of the fungicidal activities of target compounds are shown in Table 4. Overall, among the 14 new compounds, only compound 7 h showed good fungidical activities (90.5\%) against Botrytis cinereal, which was better than fluxapyroxad (63.6\%). At the same time, it had moderate inhibitory activities against Alternaria solani (50.0\%), Sclerotinia sclerotiorum (80.8\%), and Thanatephorus cucumeris $(84.8 \%)$. In addition, compounds $\mathbf{7} \mathbf{d}$ and $7 \mathbf{e}$ also showed good fungicidal activities against Botrytis cinereal $(66.7 \%, 63.1 \%)$, which was comparable to fluxapyroxad. Most compounds had moderate inhibitory activities (40-81\%) against Sclerotinia sclerotiorum, such as compounds $\mathbf{7 b}, \mathbf{7 e}, \mathbf{7 h}, \mathbf{7} \mathbf{i}, 7 \mathbf{j}, 7 \mathbf{l}$ and 7n. For FusaHum graminearum, Phytophthora capsica and Fusarium oxysporum, fluxapyroxad had almost no activities, while compounds $7 \mathbf{e}, 7 \mathbf{h}, 7 \mathrm{~m}$ had moderately strong inhibitory activities against FusaHum graminearum (40-45\%), and the other two fungi had weak activity. From Table 5, we can see 
that compound $\mathbf{7 h}$ had good inhibitory activity against Sclerotinia sclerotiorum, Botrytis cinereal and Thanatephorus cucumeris, with $\mathrm{EC}_{50}$ of $11.61,17.39$ and $17.29 \mu \mathrm{g} / \mathrm{mL}$, respectively. The SAR of the target compounds about fungicidal activities (Table 4) is that when the substituent of the benzene ring is 2-F, the inhibitory activities against the tested fungi are superior to other compounds.

Table 2. Insecticidal activities of title compounds $\mathbf{7 a - 7 n}$.

\begin{tabular}{|c|c|c|c|c|}
\hline \multirow{2}{*}{ Compounds } & \multicolumn{4}{|c|}{ Insecticidal Activities (Death Rates \%) } \\
\hline & $\begin{array}{c}\text { Concentration } \\
(\mathrm{mg} / \mathrm{L})\end{array}$ & Mythimna & $\begin{array}{c}\text { Helicoverpa } \\
\text { armigera }\end{array}$ & $\begin{array}{l}\text { Pyrausta } \\
\text { nubilalis }\end{array}$ \\
\hline $7 a$ & 500 & sepquara & 20 & 10 \\
\hline $7 \mathrm{~b}$ & 500 & 5 & 0 & 0 \\
\hline $7 \mathrm{c}$ & 500 & 20 & 15 & 5 \\
\hline $7 d$ & 500 & 30 & 10 & 15 \\
\hline $7 e$ & 500 & 40 & 25 & 20 \\
\hline $7 f$ & 500 & 25 & 20 & 15 \\
\hline $7 \mathrm{~g}$ & 500 & 25 & 15 & 10 \\
\hline $7 \mathrm{~h}$ & 500 & 15 & 10 & 5 \\
\hline $7 \mathbf{i}$ & 500 & 10 & 5 & 10 \\
\hline $7 \mathbf{j}$ & 500 & 0 & 0 & 0 \\
\hline $7 \mathrm{k}$ & 500 & 20 & 10 & 15 \\
\hline 71 & 500 & 0 & 0 & 0 \\
\hline $7 \mathrm{~m}$ & 500 & 25 & 10 & 20 \\
\hline $7 n$ & 500 & 30 & 5 & 15 \\
\hline Etoxazole & 500 & 100 & 100 & 100 \\
\hline
\end{tabular}

Table 3. Larvicidal activities of title compounds $7 \mathbf{a}-\mathbf{7 n}$.

\begin{tabular}{ccc}
\hline \multirow{2}{*}{ Compounds } & \multicolumn{2}{c}{ Larvicidal Activities (Death Rates \%) } \\
\cline { 2 - 3 } & $\begin{array}{c}\text { Concentration } \\
(\mathbf{m g} / \mathbf{L})\end{array}$ & Mosquito Larvae \\
\hline $\mathbf{7 a}$ & 10 & 100 \\
& 5 & 100 \\
$\mathbf{7 b}$ & 2 & 100 \\
$\mathbf{7 c}$ & 1 & 40 \\
$\mathbf{7 d}$ & 10 & 10 \\
$\mathbf{7 e}$ & 10 & 0 \\
$\mathbf{7 f}$ & 10 & 0 \\
$\mathbf{7 g}$ & 10 & 0 \\
$\mathbf{7 h}$ & 10 & 100 \\
$\mathbf{7 i}$ & 5 & 55 \\
$7 \mathbf{j}$ & 10 & 5 \\
$\mathbf{7 k}$ & 10 & 20 \\
$\mathbf{7 1}$ & 10 & 15 \\
$\mathbf{7 m}$ & 10 & 25 \\
$\mathbf{7 n}$ & 10 & 30 \\
$\mathbf{7 n}$ & 10 & 0 \\
Etoxazole & 10 & 45 \\
& 10 & 25 \\
& 10 & 100 \\
\hline
\end{tabular}


Table 4. Fungicidal activities of title compounds $7 \mathbf{a}-7 \mathbf{n}$ at $50 \mathrm{mg} / \mathrm{L}$.

\begin{tabular}{|c|c|c|c|c|c|c|c|c|}
\hline \multirow{2}{*}{ Compounds } & \multicolumn{8}{|c|}{ Fungicidal Activities (Inhibition Rate \%) } \\
\hline & $A S$ & $F G$ & $C A$ & $P C$ & SS & $B C$ & $T C$ & $F O$ \\
\hline $7 a$ & 44.4 & 36.1 & 6.7 & 8.3 & 30.8 & 33.3 & 50.0 & 4.5 \\
\hline $7 \mathrm{~b}$ & 16.7 & 27.8 & 6.7 & 25.0 & 46.2 & 19.0 & 30.3 & 4.5 \\
\hline $7 \mathrm{c}$ & 16.7 & 27.8 & 6.7 & 25.0 & 30.8 & 33.3 & 27.3 & 4.5 \\
\hline $7 d$ & 27.8 & 36.1 & 13.3 & 8.3 & 30.8 & 66.7 & 40.9 & 22.7 \\
\hline $7 e$ & 30.8 & 40.4 & 6.7 & 28.7 & 41.4 & 63.1 & 44.5 & 31.6 \\
\hline $7 \mathrm{f}$ & 11.1 & 22.2 & 18.2 & 33.3 & 15.4 & 28.6 & 15.2 & 13.6 \\
\hline $7 \mathrm{~g}$ & 33.3 & 25.0 & 33.3 & 8.3 & 15.4 & 23.8 & 50.0 & 9.1 \\
\hline $7 \mathrm{~h}$ & 50.0 & 44.4 & 40.0 & 8.3 & 80.8 & 90.5 & 84.8 & 22.7 \\
\hline $7 \mathbf{i}$ & 27.8 & 36.1 & 13.3 & 16.7 & 42.3 & 47.6 & 25.8 & 18.2 \\
\hline $7 \mathbf{j}$ & 27.8 & 22.2 & 6.7 & 16.7 & 73.1 & 38.1 & 54.5 & 22.7 \\
\hline $7 \mathrm{k}$ & 11.1 & 30.6 & 13.3 & 8.3 & 15.4 & 23.8 & 15.2 & 18.2 \\
\hline 71 & 16.7 & 5.6 & 13.3 & 16.7 & 46.2 & 33.3 & 22.7 & 18.2 \\
\hline $7 \mathrm{~m}$ & 38.9 & 41.7 & 26.7 & 25.0 & 38.5 & 38.1 & 60.6 & 13.6 \\
\hline $7 \mathbf{n}$ & 22.2 & 22.2 & 6.7 & 8.3 & 46.2 & 9.5 & 22.7 & 9.1 \\
\hline Fluxapyroxad & 88.9 & 30.3 & 100 & 38.1 & 96.4 & 63.6 & 88.4 & 44.4 \\
\hline
\end{tabular}

Note: Alternaria solani (AS), FusaHum graminearum (FG), Cercospora arachidicola (CA), Phytophthora capsica (PC), Sclerotinia sclerotiorum (SS), Botrytis cinereal (BC), Thanatephorus cucumeris (TC), Fusarium oxysporum (FO). All the data were determined three times.

Table 5. $\mathrm{EC}_{50}$ of compounds $7 \mathrm{~h}$ to Sclerotinia sclerotiorum (SS), Botrytis cinereal (BC), Thanatephorus cucumeris (TC).

\begin{tabular}{cccc}
\hline Fungus & $\mathbf{y}=\mathbf{a}+\mathbf{b x}$ & $\mathbf{r}^{\mathbf{2}}$ & $\mathbf{E C}_{\mathbf{5 0}} /\left(\boldsymbol{\mu g} \cdot \mathbf{m} \mathbf{L}^{-\mathbf{1}}\right)$ \\
\hline$S S$ & $\mathrm{y}=1.5805 \mathrm{x}+3.3168$ & 0.9836 & 11.61 \\
$B C$ & $\mathrm{y}=2.1065 \mathrm{x}+2.3871$ & 0.9758 & 17.39 \\
$T C$ & $\mathrm{y}=1.8992 \mathrm{x}+2.6489$ & 0.9815 & 17.29 \\
\hline
\end{tabular}

\section{Experimental Section}

\subsection{General Information}

Melting points were determined using an X-4 digital microscopic melting point detector (Taike, Beijing, China) and the thermometer was uncorrected. ${ }^{1} \mathrm{H}-\mathrm{NMR}$ and ${ }^{13} \mathrm{C}-\mathrm{NMR}$ spectra were measured on NMR spectrometer (Bruker $500 \mathrm{MHz}$, Fallanden, Switzerland); High-resolution electrospray mass spectra (HR-ESI-MS) were determined using an UPLC H-CLASS/QTOF G2-XS mass spectrometer (Waters, Milford, MA, USA). All the reagents and solvents were in analytical purity. The characterisation data for all synthesised compounds are provided in the Supporting Information file.

\subsection{Synthesis}

\subsubsection{Methyl 2-chloro-5-iodobenzoate 2}

2-chloro-5-iodobenzoic acid 1 (2.8 g, $0.01 \mathrm{~mol})$, methanol (50 mL) and $\mathrm{H}_{2} \mathrm{SO} 4(0.5 \mathrm{~mL})$ were added to a three-necked flask and reacted at reflux for about $8 \mathrm{~h}$. After the mixture was cooled to room temperature, methanol was removed under reduced pressure. Then, EtOAc $(50 \mathrm{~mL})$ was added and the $\mathrm{pH}$ adjusted to $7-8$ by using $\mathrm{NaHCO}_{3}$. The organic layer was dried with $\mathrm{Na}_{2} \mathrm{SO}_{4}$. The solvent was removed to give $2.71 \mathrm{~g}$ creamy white solid. Yield: 92\%, m.p. $40 \sim 42{ }^{\circ} \mathrm{C} ;{ }^{1} \mathrm{H}-\mathrm{NMR}(500 \mathrm{MHz}$, Chloroform- $d$ ) $\delta 8.14(\mathrm{~d}, J=2.0 \mathrm{~Hz}, 1 \mathrm{H}), 7.72(\mathrm{dd}, J=8.5,2.5 \mathrm{~Hz}, 1 \mathrm{H}), 7.19(\mathrm{~d}, J=8.0 \mathrm{~Hz}, 1 \mathrm{H}), 3.94$ $(\mathrm{s}, 3 \mathrm{H})$. 


\subsubsection{Methyl 2-chloro-5-cyanobenzoate 3}

Methyl 2-chloro-5-iodobenzoate 2 (0.40 g, $1.3 \mathrm{mmol}), \mathrm{CuCN}(0.18 \mathrm{~g}, 2.0 \mathrm{mmol})$, L-proline $(0.15 \mathrm{~g}$, $1.3 \mathrm{mmol})$ and DMF (15 mL) were added to a three-necked flask. After being dissolved, the mixture was heated to $70{ }^{\circ} \mathrm{C}$ and reacted for $2 \mathrm{~h}$. Then, the temperature was heated to $100{ }^{\circ} \mathrm{C}$. The reaction was complete after $9 \mathrm{~h}$. After the mixture was cooled to room temperature, it was filtered with diatomite. The filtrate was extracted by water $(100 \mathrm{~mL})$ and EtOAc $(100 \mathrm{~mL})$. The organic layer was washed with water $(50 \mathrm{~mL} \times 3)$ and then dried with $\mathrm{MgSO}_{4}$. EtOAc was removed under reduced pressure and 0.20 g yellow solid was obtained. Yield: $79 \%$, m.p. $102-104{ }^{\circ} \mathrm{C}\left([27], 100-103{ }^{\circ} \mathrm{C}\right)$.

\subsubsection{Methyl 2-chloro-5-( $N^{\prime}$-hydroxycarbamimidoyl)benzoate 4}

Methyl 2-chloro-5-cyanobenzoate $3(1.4 \mathrm{~g}, 7.2 \mathrm{mmol})$ was added to a three-necked flask and dissolved by ethanol $(45 \mathrm{~mL})$. Stirring was started at room temperature, and then hydroxylamine hydrochloride $(0.75 \mathrm{~g})$ and triethylamine $(1.1 \mathrm{~g})$ were gradually added. The mixture was stirred for $3 \mathrm{~h}$. Then the solvent was removed under reduced pressure and the remnant was dissolved in EtOAc $(50 \mathrm{~mL})$ and saturated saline $(50 \mathrm{~mL})$. The organic layer was dried with $\mathrm{Na}_{2} \mathrm{SO}_{4}$ and evaporated, to give $1.5 \mathrm{~g}$ light yellow solid. Yield: $90 \%$, m.p. $108-111{ }^{\circ} \mathrm{C} ;{ }^{1} \mathrm{H}-\mathrm{NMR}\left(500 \mathrm{MHz}, \mathrm{DMSO}-d_{6}\right) \delta 9.87(\mathrm{~s}, 1 \mathrm{H})$, $8.13(\mathrm{~d}, J=2.0 \mathrm{~Hz}, 1 \mathrm{H}), 7.85(\mathrm{dd}, J=8.5,2.0 \mathrm{~Hz}, 1 \mathrm{H}), 7.59(\mathrm{~d}, J=8.5 \mathrm{~Hz}, 1 \mathrm{H}), 5.98(\mathrm{~s}, 2 \mathrm{H}), 3.87(\mathrm{~s}, 3 \mathrm{H})$.

\subsubsection{Methyl 2-chloro-5-(5-(3,6-dichloropyridin-2-yl)-1,2,4-oxadiazol-3-yl)benzoate 5}

3,6-dichloropicolinic acid $(1.1 \mathrm{~g}, 5 \mathrm{mmol})$ and $\mathrm{SOCl}_{2}(20 \mathrm{~mL})$ were added to a round bottom flask. The mixture was stirred and refluxed for $2 \mathrm{~h}$. Then, $\mathrm{SOCl}_{2}$ was removed under reduced pressure to give 3,6-dichloropicolinoyl chloride.

Intermediate $4(1.1 \mathrm{~g}, 5 \mathrm{mmol})$, triethylamine $(1.2 \mathrm{~g}, 5 \mathrm{mmol})$ and dry toluene $(100 \mathrm{~mL})$ were added to a three-necked flask. The mixture was stirred at $0{ }^{\circ} \mathrm{C}$ for $2 \mathrm{~h}$. After that, the prepared 3,6-dichloropicolinoyl chloride (dissolved by $30 \mathrm{~mL}$ dry toluene) was dropped into the flask. The mixture continued to be stirred for $1 \mathrm{~h}$ at $0{ }^{\circ} \mathrm{C}$. Then, the temperature was increased to reflux for $2 \mathrm{~h}$. When the mixture was cooled to room temperature, it was washed by saturated sodium chloride solution $(150 \mathrm{~mL} \times 3)$. The organic layer was dried by $\mathrm{Na}_{2} \mathrm{SO}_{4}$ and removed under reduced pressure to give $1.5 \mathrm{~g}$ yellow solid. Yield: $78 \%$, m.p. $148-152{ }^{\circ} \mathrm{C} ;{ }^{1} \mathrm{H}-\mathrm{NMR}(500 \mathrm{MHz}$, Chloroform-d) $\delta 8.65(\mathrm{~s}, 1 \mathrm{H})$, 8.32-8.16 (m, 1H), $7.92(\mathrm{~d}, J=8.5 \mathrm{~Hz}, 1 \mathrm{H}), 7.61(\mathrm{~d}, J=8.5 \mathrm{~Hz}, 1 \mathrm{H}), 7.54(\mathrm{~d}, J=8.5 \mathrm{~Hz}, 1 \mathrm{H}), 3.98(\mathrm{~s}, 3 \mathrm{H})$.

\subsubsection{2-chloro-5-(5-(3,6-dichloropyridin-2-yl)-1,2,4-oxadiazol-3-yl)benzoic acid 6}

Intermediate $5(0.8 \mathrm{~g}, 2.0 \mathrm{mmol})$ and THF $(40 \mathrm{~mL})$ were added to a three-necked flask. After being dissolved, $30 \% \mathrm{NaOH}(5 \mathrm{~mL})$ was also added to the flask and refluxed for $2 \mathrm{~h}$. After the mixture was cooled to room temperature, the solvent was removed. Then, the $\mathrm{pH}$ was adjusted to 2-3 with $\mathrm{HCl}$ and $0.7 \mathrm{~g}$ white solid precipitate was obtained. Yield: $94 \%$, m.p. $203-204{ }^{\circ} \mathrm{C} ;{ }^{1} \mathrm{H}-\mathrm{NMR}\left(500 \mathrm{MHz}, \mathrm{DMSO}-d_{6}\right)$ $\delta 13.64(\mathrm{~s}, 1 \mathrm{H}), 8.45(\mathrm{~d}, J=2.1 \mathrm{~Hz}, 1 \mathrm{H}), 8.34(\mathrm{~s}, 1 \mathrm{H}), 8.26-8.17(\mathrm{~m}, 1 \mathrm{H}), 7.91(\mathrm{~d}, J=8.6 \mathrm{~Hz}, 1 \mathrm{H}), 7.80(\mathrm{~d}$, $J=8.3 \mathrm{~Hz}, 1 \mathrm{H})$.

\subsubsection{Preparation of Target Compound 7}

Intermediate $6(4.1 \mathrm{~g}, 11 \mathrm{mmol})$, triethylamine $(0.2 \mathrm{~g}), \mathrm{DCM}(30 \mathrm{~mL})$ and EDCI $(0.3 \mathrm{~g})$ were added to a three-necked flask. Substituted aniline $(12 \mathrm{mmol})$ was stirred and dropped into the flask at $0{ }^{\circ} \mathrm{C}$. TLC was used to track reaction progress. At last, target compound 7 was obtained by using the method of column chromatography separation.

2-chloro-5-(5-(3,6-dichloropyridin-2-yl)-1,2,4-oxadiazol-3-yl)-N-phenylbenzamide 7a. Yellow solid, yield 64\%, m.p. 234-238 ${ }^{\circ} \mathrm{C},{ }^{1} \mathrm{H}-\mathrm{NMR}\left(500 \mathrm{MHz}, \mathrm{DMSO}-\mathrm{d}_{6}\right) \delta 10.70(\mathrm{~s}, 1 \mathrm{H}), 8.36$ (d, J = 8.5 Hz, 1H), 8.25-8.18 $(\mathrm{m}, 2 \mathrm{H}), 7.92(\mathrm{~d}, J=9.0 \mathrm{~Hz}, 1 \mathrm{H}), 7.85(\mathrm{~d}, J=8.0 \mathrm{~Hz}, 1 \mathrm{H}), 7.73(\mathrm{~d}, J=8.0 \mathrm{~Hz}, 2 \mathrm{H}), 7.38(\mathrm{t}, J=7.5 \mathrm{~Hz}$, 2H), 7.19-7.09 (m, 1H); ${ }^{13} \mathrm{C}-\mathrm{NMR}\left(500 \mathrm{MHz}, \mathrm{DMSO}-d_{6}\right) \delta 171.75,167.27,163.91,148.60,143.34,139.71$, 
138.69, 137.84, 133.65, 131.28, 131.10, 129.52, 129.27, 128.88, 127.32, 124.77, 124.13, 119.78; HRMS calcd. for $\mathrm{C}_{20} \mathrm{H}_{11} \mathrm{Cl}_{3} \mathrm{~N}_{4} \mathrm{O}_{2}[\mathrm{M}+\mathrm{Na}]^{+} 466.9840$, found 466.9840 .

2-chloro-5-(5-(3,6-dichloropyridin-2-yl)-1,2,4-oxadiazol-3-yl)-N-(o-tolyl)benzamide $7 \mathbf{b}$. Yellow solid, yield 77\%, m.p. $220-223{ }^{\circ} \mathrm{C},{ }^{1} \mathrm{H}-\mathrm{NMR}\left(500 \mathrm{MHz}, \mathrm{DMSO}-\mathrm{d}_{6}\right) \delta 10.60(\mathrm{~s}, 1 \mathrm{H}), 8.34(\mathrm{~d}, J=9.0 \mathrm{~Hz}, 1 \mathrm{H})$, $8.21(\mathrm{~d}, J=7.0 \mathrm{~Hz}, 2 \mathrm{H}), 7.91(\mathrm{~d}, J=9.0 \mathrm{~Hz}, 1 \mathrm{H}), 7.83(\mathrm{~d}, J=9.0 \mathrm{~Hz}, 1 \mathrm{H}), 7.61(\mathrm{~d}, J=8.5 \mathrm{~Hz}, 2 \mathrm{H}), 7.17(\mathrm{~d}$, $J=8.5 \mathrm{~Hz}, 2 \mathrm{H}), 2.29(\mathrm{~s}, 3 \mathrm{H}) ;{ }^{13} \mathrm{C}-\mathrm{NMR}\left(500 \mathrm{MHz}, \mathrm{DMSO}-d_{6}\right) \delta 171.72,167.26,163.68,148.59,143.31$, 139.68, 137.90, 136.19, 133.65, 133.12, 131.25, 131.07, 129.43, 129.24, 129.21, 127.31, 124.73, 119.76, 20.52; HRMS calcd. for $\mathrm{C}_{21} \mathrm{H}_{13} \mathrm{Cl}_{3} \mathrm{~N}_{4} \mathrm{O}_{2}[\mathrm{M}+\mathrm{Na}]^{+} 480.9996$, found 480.9995 .

2-chloro-5-(5-(3,6-dichloropyridin-2-yl)-1,2,4-oxadiazol-3-yl)-N-(m-tolyl)benzamide 7c. Yellow solid, yield 74\%, m.p. $260-261{ }^{\circ} \mathrm{C},{ }^{1} \mathrm{H}-\mathrm{NMR}\left(500 \mathrm{MHz}, \mathrm{DMSO}-d_{6}\right) \delta 10.69(\mathrm{~s}, 1 \mathrm{H}), 8.35(\mathrm{~d}, J=8.5 \mathrm{~Hz}, 1 \mathrm{H})$, $8.25-8.17(\mathrm{~m}, 2 \mathrm{H}), 7.92(\mathrm{~d}, J=9.0 \mathrm{~Hz}, 1 \mathrm{H}), 7.83(\mathrm{~d}, J=9.0 \mathrm{~Hz}, 1 \mathrm{H}), 7.60(\mathrm{~s}, 1 \mathrm{H}), 7.51(\mathrm{~d}, J=8.5 \mathrm{~Hz}, 1 \mathrm{H})$, $7.25(\mathrm{t}, J=8.0 \mathrm{~Hz}, 1 \mathrm{H}), 6.96(\mathrm{~d}, J=7.5 \mathrm{~Hz}, 1 \mathrm{H}), 2.32(\mathrm{~s}, 3 \mathrm{H}) ;{ }^{13} \mathrm{C}-\mathrm{NMR}\left(500 \mathrm{MHz}, \mathrm{DMSO}-d_{6}\right) \delta 171.73$, 167.27, 163.88, 148.60, 143.34, 139.70, 138.63, 138.10, 137.90, 133.65, 131.27, 131.08, 129.47, 129.27, 128.69, 127.30, 124.80, 124.74, 120.29, 117.01, 21.22; HRMS calcd. for $\mathrm{C}_{21} \mathrm{H}_{13} \mathrm{Cl}_{3} \mathrm{~N}_{4} \mathrm{O}_{2}[\mathrm{M}+\mathrm{Na}]^{+} 480.9996$, found 480.9998 .

2-chloro-5-(5-(3,6-dichloropyridin-2-yl)-1,2,4-oxadiazol-3-yl)-N-(p-tolyl)benzamide 7d. White solid, yield $68 \%$, m.p. $230-232{ }^{\circ} \mathrm{C},{ }^{1} \mathrm{H}-\mathrm{NMR}\left(500 \mathrm{MHz}, \mathrm{DMSO}-d_{6}\right) \delta 10.19(\mathrm{~s}, 1 \mathrm{H}), 8.36(\mathrm{~d}, J=10 \mathrm{~Hz}, 1 \mathrm{H}), 8.27$ $(\mathrm{d}, J=1.9 \mathrm{~Hz}, 1 \mathrm{H}), 8.24-8.18(\mathrm{~m}, 1 \mathrm{H}), 7.93(\mathrm{~d}, J=8.5 \mathrm{~Hz}, 1 \mathrm{H}), 7.84(\mathrm{~d}, J=8.4 \mathrm{~Hz}, 1 \mathrm{H}), 7.50(\mathrm{~d}, J=7.7 \mathrm{~Hz}$, 1H), $7.29(\mathrm{~d}, J=7.3 \mathrm{~Hz}, 1 \mathrm{H}), 7.25(\mathrm{t}, J=7.3 \mathrm{~Hz}, 2 \mathrm{H}), 7.20-7.16(\mathrm{~m}, 1 \mathrm{H}), 2.32(\mathrm{~s}, 3 \mathrm{H}) ;{ }^{13} \mathrm{C}-\mathrm{NMR}(500 \mathrm{MHz}$, DMSO- $\left.d_{6}\right) \delta 171.76,167.31,164.23,148.63,143.34,139.74,137.98,135.55,133.66,132.97,131.28,131.08$, $129.43,129.28,127.40,126.19,126.12,126.04,124.74,18.01$; HRMS calcd. for $\mathrm{C}_{21} \mathrm{H}_{13} \mathrm{Cl}_{3} \mathrm{~N}_{4} \mathrm{O}_{2}[\mathrm{M}+\mathrm{Na}]^{+}$ 480.9996, found 480.9995 .

N-(4-(tert-butyl)phenyl)-2-chloro-5-(5-(3,6-dichloropyridin-2-yl)-1,2,4-oxadiazol-3-yl)benzamide 7e. Grey solid, yield 69\%, m.p. $208-210{ }^{\circ} \mathrm{C},{ }^{1} \mathrm{H}-\mathrm{NMR}\left(500 \mathrm{MHz}, \mathrm{DMSO}-d_{6}\right) \delta 10.64(\mathrm{~s}, 1 \mathrm{H}), 8.35(\mathrm{~d}, J=9.0 \mathrm{~Hz}, 1 \mathrm{H})$, 8.23-8.18 (m, 2H), $7.92(\mathrm{~d}, J=8.5 \mathrm{~Hz}, 1 \mathrm{H}), 7.83(\mathrm{~d}, J=9.0 \mathrm{~Hz}, 1 \mathrm{H}), 7.64(\mathrm{~d}, J=8.5 \mathrm{~Hz}, 2 \mathrm{H}), 7.38(\mathrm{~d}$, $J=8.5 \mathrm{~Hz}, 2 \mathrm{H}), 1.28(\mathrm{~s}, 3 \mathrm{H}) ;{ }^{13} \mathrm{C}-\mathrm{NMR}\left(500 \mathrm{MHz}, \mathrm{DMSO}-d_{6}\right) \delta 171.70,167.25,163.71,148.58,146.49$, 143.31, 139.67, 137.91, 136.12, 133.65, 131.24, 131.05, 129.41, 129.24, 127.28, 126.44, 125.44, 124.71, 119.54, 34.09, 31.18; HRMS calcd. for $\mathrm{C}_{24} \mathrm{H}_{19} \mathrm{Cl}_{3} \mathrm{~N}_{4} \mathrm{O}_{2}[\mathrm{M}+\mathrm{Na}]^{+}$523.0466, found 523.0466.

2-chloro-5-(5-(3,6-dichloropyridin-2-yl)-1,2,4-oxadiazol-3-yl)-N-(3-(trifluoromethyl)phenyl)benzamide $7 \mathbf{f}$. White solid, yield 77\%, m.p. 191-195 ${ }^{\circ} \mathrm{C}$, H-NMR (500 MHz, DMSO- $\left.d_{6}\right) \delta 11.02(\mathrm{~s}, 1 \mathrm{H}), 8.30(\mathrm{~d}, J=8.5$ $\mathrm{Hz}, 1 \mathrm{H}), 8.25(\mathrm{~s}, 1 \mathrm{H}), 8.19(\mathrm{~s}, 2 \mathrm{H}), 7.87(\mathrm{~d}, J=8.5 \mathrm{~Hz}, 2 \mathrm{H}), 7.81(\mathrm{~d}, J=8.5 \mathrm{~Hz}, 1 \mathrm{H}), 7.58(\mathrm{t}, 1 \mathrm{H}), 7.45(\mathrm{~d}$, $J=7.5 \mathrm{~Hz}, 1 \mathrm{H}) ;{ }^{13} \mathrm{C}-\mathrm{NMR}\left(500 \mathrm{MHz}\right.$, DMSO- $\left.d_{6}\right) \delta 171.76,167.24,164.35,148.61,143.32,139.67,139.42$, 137.30, 133.66, 131.27, 131.15, 130.19, 129.81, 129.26, 127.45, 124.84, 123.37, 120.48, 115.84; HRMS calcd. for $\mathrm{C}_{21} \mathrm{H}_{10} \mathrm{Cl}_{3} \mathrm{~F}_{3} \mathrm{~N}_{4} \mathrm{O}_{2}[\mathrm{M}+\mathrm{Na}]^{+}$534.9714, found 534.9717 .

2-chloro-5-(5-(3,6-dichloropyridin-2-yl)-1,2,4-oxadiazol-3-yl)-N-(2,4-dimethylphenyl)benzamide $\quad \mathbf{7 g}$. Yellow solid, yield 60\%, m.p. $225-226{ }^{\circ} \mathrm{C},{ }^{1} \mathrm{H}-\mathrm{NMR}\left(500 \mathrm{MHz}\right.$, DMSO- $\left.d_{6}\right) \delta 10.09$ (s, $\left.1 \mathrm{H}\right), 8.37$ (d, $J=8.5$ $\mathrm{Hz}, 1 \mathrm{H}), 8.25(\mathrm{~d}, J=2.0 \mathrm{~Hz}, 1 \mathrm{H}), 8.22(\mathrm{~d}, J=2.0 \mathrm{~Hz}, 1 \mathrm{H}), 8.20(\mathrm{~d}, J=2.0 \mathrm{~Hz}, 1 \mathrm{H}), 7.94(\mathrm{~d}, J=8.5 \mathrm{~Hz}, 1 \mathrm{H})$, $7.84(\mathrm{~d}, J=8.0 \mathrm{~Hz}, 1 \mathrm{H}), 7.35(\mathrm{~d}, J=8.0 \mathrm{~Hz}, 1 \mathrm{H}), 7.09(\mathrm{~s}, 1 \mathrm{H}), 7.04(\mathrm{~d}, J=8.0 \mathrm{~Hz}, 1 \mathrm{H}), 2.29(\mathrm{~s}, 3 \mathrm{H}), 2.27(\mathrm{~s}$, $3 \mathrm{H}) ;{ }^{13} \mathrm{C}-\mathrm{NMR}(500 \mathrm{MHz}$, DMSO-d 6 ) $\delta 171.76,167.31,164.23,148.62,143.34,139.75,138.05,135.33$, 133.65, 132.94, 132.83, 131.27, 131.07, 130.98, 129.37, 129.28, 127.38, 126.61, 125.99, 124.71, 20.56, 17.92; HRMS calcd. for $\mathrm{C}_{22} \mathrm{H}_{15} \mathrm{Cl}_{3} \mathrm{~N}_{4} \mathrm{O}_{2}[\mathrm{M}+\mathrm{Na}]^{+} 495.0153$, found 495.0154 .

2-chloro-5-(5-(3,6-dichloropyridin-2-yl)-1,2,4-oxadiazol-3-yl)-N-(2-fluorophenyl)benzamide $7 \mathrm{~h}$. Yellow solid, yield 60\%, m.p. $203-207^{\circ} \mathrm{C},{ }^{1} \mathrm{H}-\mathrm{NMR}\left(500 \mathrm{MHz}, \mathrm{DMSO}-d_{6}\right) \delta 10.57(\mathrm{~s}, 1 \mathrm{H}), 8.35(\mathrm{~d}, J=8.5 \mathrm{~Hz}$, $1 \mathrm{H}), 8.27-8.19(\mathrm{~m}, 2 \mathrm{H}), 7.92(\mathrm{~d}, J=8.5 \mathrm{~Hz}, 1 \mathrm{H}), 7.90-7.86(\mathrm{~m}, 1 \mathrm{H}), 7.83(\mathrm{~d}, J=8.0 \mathrm{~Hz}, 1 \mathrm{H}), 7.35-7.21$ (m, 3H); ${ }^{13} \mathrm{C}-\mathrm{NMR}\left(500 \mathrm{MHz}\right.$, DMSO- $\left.d_{6}\right) \delta 171.78,167.30,164.41,148.65,143.34,139.73,137.35,133.79$, 131.29, 131.10, 129.65, 129.29, 127.54, 126.81 (d, $J=7.5 \mathrm{~Hz}), 125.70,125.28,125.19,124.69,124.51(\mathrm{~d}$, $J=3.0 \mathrm{~Hz}), 115.88(\mathrm{~d}, J=19.0 \mathrm{~Hz})$; HRMS calcd. for $\mathrm{C}_{20} \mathrm{H}_{10} \mathrm{Cl}_{3} \mathrm{FN}_{4} \mathrm{O}_{2}[\mathrm{M}+\mathrm{Na}]^{+}$484.9746, found 484.9747.

2-chloro-5-(5-(3,6-dichloropyridin-2-yl)-1,2,4-oxadiazol-3-yl)-N-(3-fluorophenyl)benzamide 7i. Yellow solid, yield 62\%, m.p. $228-230{ }^{\circ} \mathrm{C},{ }^{1} \mathrm{H}-\mathrm{NMR}\left(500 \mathrm{MHz}\right.$, DMSO- $\left.d_{6}\right) \delta 10.91(\mathrm{~s}, 1 \mathrm{H}), 8.35(\mathrm{~d}, J=9.0 \mathrm{~Hz}$, 
$1 \mathrm{H}), 8.30-8.20(\mathrm{~m}, 2 \mathrm{H}), 7.92(\mathrm{~d}, J=8.5 \mathrm{~Hz}, 1 \mathrm{H}), 7.86(\mathrm{~d}, J=8.5 \mathrm{~Hz}, 1 \mathrm{H}), 7.70(\mathrm{~d}, J=11.5 \mathrm{~Hz}, 1 \mathrm{H})$, 7.53-7.36 (m, 2H), 7.03-6.92 (m, 1H); ${ }^{13} \mathrm{C}-\mathrm{NMR}\left(500 \mathrm{MHz}, \mathrm{DMSO}-d_{6}\right) \delta 171.76,167.23,164.15,163.10$, $161.18,148.61,143.33,139.69,137.46,133.62,131.21(\mathrm{~d}, J=16.0 \mathrm{~Hz}), 130.58(\mathrm{~d}, J=9.5 \mathrm{~Hz}), 129.72,129.27$, $127.37,124.82,115.55,110.64(\mathrm{~d}, J=21.0 \mathrm{~Hz}), 106.68,106.47$; $\mathrm{HRMS}$ calcd. for $\mathrm{C}_{20} \mathrm{H}_{10} \mathrm{Cl}_{3} \mathrm{FN}_{4} \mathrm{O}_{2}[\mathrm{M}+$ $\mathrm{Na}]^{+} 484.9746$, found 484.9747 .

2-chloro-5-(5-(3,6-dichloropyridin-2-yl)-1,2,4-oxadiazol-3-yl)-N-(4-fluorophenyl)benzamide 7j. Yellow solid, yield 63\%, m.p. $248-251{ }^{\circ} \mathrm{C},{ }^{1} \mathrm{H}-\mathrm{NMR}\left(500 \mathrm{MHz}\right.$, DMSO- $\left.d_{6}\right) \delta 10.77(\mathrm{~s}, 1 \mathrm{H}), 8.36(\mathrm{~d}, J=8.5 \mathrm{~Hz}$, $1 \mathrm{H}), 8.26-8.19(\mathrm{~m}, 2 \mathrm{H}), 7.93(\mathrm{~d}, J=9.0 \mathrm{~Hz}, 1 \mathrm{H}), 7.85(\mathrm{~d}, J=8.5 \mathrm{~Hz}, 1 \mathrm{H}), 7.79-7.72(\mathrm{~m}, 2 \mathrm{H}), 7.22(\mathrm{t}, J=9.0$ $\mathrm{Hz}, 2 \mathrm{H}) ;{ }^{13} \mathrm{C}-\mathrm{NMR}\left(500 \mathrm{MHz}\right.$, DMSO- $\left.d_{6}\right) \delta 171.79,167.29,163.85,148.64,143.36,139.73,137.70,133.68$, 131.30, 131.16, 129.62, 129.30, 127.37, 124.82, 121.67 (d, J = 7.9 Hz), 115.61, 115.44; HRMS calcd. for $\mathrm{C}_{20} \mathrm{H}_{10} \mathrm{Cl}_{3} \mathrm{FN}_{4} \mathrm{O}_{2}[\mathrm{M}+\mathrm{Na}]^{+} 484.9746$, found 484.9749 .

2-chloro-N-(2-chlorophenyl)-5-(5-(3,6-dichloropyridin-2-yl)-1,2,4-oxadiazol-3-yl)benzamide 7k. Yellow solid, yield 65\%, m.p. 195-198 ${ }^{\circ} \mathrm{C},{ }^{1} \mathrm{H}-\mathrm{NMR}\left(500 \mathrm{MHz}\right.$, DMSO- $\left.d_{6}\right) \delta 10.47(\mathrm{~s}, 1 \mathrm{H}), 8.36(\mathrm{~d}, J=9.0 \mathrm{~Hz}$, $1 \mathrm{H}), 8.30(\mathrm{~s}, 1 \mathrm{H}), 8.23(\mathrm{~d}, J=8.5 \mathrm{~Hz}, 1 \mathrm{H}), 7.93(\mathrm{~d}, J=9.0 \mathrm{~Hz}, 1 \mathrm{H}), 7.84(\mathrm{~d}, J=8.5 \mathrm{~Hz}, 1 \mathrm{H}), 7.75(\mathrm{~d}, J=8.0$ $\mathrm{Hz}, 1 \mathrm{H}), 7.57(\mathrm{~d}, J=8.0 \mathrm{~Hz}, 1 \mathrm{H}), 7.42(\mathrm{t}, J=7.5 \mathrm{~Hz}, 1 \mathrm{H}), 7.32(\mathrm{t}, J=7.5 \mathrm{~Hz}, 1 \mathrm{H}) ;{ }^{13} \mathrm{C}-\mathrm{NMR}(500 \mathrm{MHz}$, DMSO- $\left.d_{6}\right) \delta 171.76,167.27,164.43,148.64,143.34,139.73,137.27,134.23,133.81,131.27,131.15,129.75$, $129.66,129.28,128.60,127.87,127.70,127.61,127.58,124.68$; HRMS calcd. for $\mathrm{C}_{20} \mathrm{H}_{10} \mathrm{Cl}_{4} \mathrm{~N}_{4} \mathrm{O}_{2}[\mathrm{M}+\mathrm{Na}]^{+}$ 500.9450 , found 500.9452 .

2-chloro-N-(3-chlorophenyl)-5-(5-(3,6-dichloropyridin-2-yl)-1,2,4-oxadiazol-3-yl)benzamide 71. Yellow solid, yield 65\%, m.p. $214-218{ }^{\circ} \mathrm{C},{ }^{1} \mathrm{H}-\mathrm{NMR}\left(500 \mathrm{MHz}, \mathrm{DMSO}-d_{6}\right) \delta 10.90(\mathrm{~s}, 1 \mathrm{H}), 8.35(\mathrm{~d}, J=9.0 \mathrm{~Hz}$, $1 \mathrm{H}), 8.26(\mathrm{~d}, J=2.0 \mathrm{~Hz}, 1 \mathrm{H}), 8.23(\mathrm{dd}, J=8.5,2.0 \mathrm{~Hz}, 1 \mathrm{H}), 7.96-7.90(\mathrm{~m}, 2 \mathrm{H}), 7.85(\mathrm{~d}, J=8.5 \mathrm{~Hz}, 1 \mathrm{H})$, $7.60(\mathrm{~d}, J=9.0 \mathrm{~Hz}, 1 \mathrm{H}), 7.41(\mathrm{t}, J=8.5 \mathrm{~Hz}, 1 \mathrm{H}), 7.22(\mathrm{~s}, 1 \mathrm{H}) ;{ }^{13} \mathrm{C}-\mathrm{NMR}\left(126 \mathrm{MHz}\right.$, DMSO- $\left.d_{6}\right) \delta 171.79$, 167.27, 164.22, 148.64, 143.36, 140.09, 139.71, 133.67, 133.23, 131.31, 131.18, 130.66, 129.78, 129.30, 127.42, 124.86, 123.91, 119.30, 118.24; HRMS calcd. for $\mathrm{C}_{20} \mathrm{H}_{10} \mathrm{Cl}_{4} \mathrm{~N}_{4} \mathrm{O}_{2}[\mathrm{M}+\mathrm{Na}]^{+}$500.9450, found 500.9454.

2-chloro-N-(4-chlorophenyl)-5-(5-(3,6-dichloropyridin-2-yl)-1,2,4-oxadiazol-3-yl)benzamide $7 \mathbf{m}$. Yellow solid, yield 67\%, m.p. $242-243{ }^{\circ} \mathrm{C} .{ }^{1} \mathrm{H}-\mathrm{NMR}\left(500 \mathrm{MHz}, \mathrm{DMSO}-d_{6}\right) \delta 10.85(\mathrm{~s}, 1 \mathrm{H}), 8.35(\mathrm{~d}, J=8.5 \mathrm{~Hz}$, $1 \mathrm{H}), 8.28-8.18(\mathrm{~m}, 2 \mathrm{H}), 7.92(\mathrm{~d}, J=9.0 \mathrm{~Hz}, 1 \mathrm{H}), 7.85(\mathrm{~d}, J=8.5 \mathrm{~Hz}, 1 \mathrm{H}), 7.76(\mathrm{~d}, J=9.0 \mathrm{~Hz}, 2 \mathrm{H}), 7.44(\mathrm{~d}$, $J=9.0 \mathrm{~Hz}, 2 \mathrm{H}) ;{ }^{13} \mathrm{C}-\mathrm{NMR}\left(126 \mathrm{MHz}, \mathrm{DMSO}-d_{6}\right) \delta 171.79,167.28,164.03,148.64,143.36,139.72,137.64$, $137.57,133.67,131.31,131.17,129.71,129.30,128.84,127.80,127.39,124.84,121.39$; HRMS calcd. for $\mathrm{C}_{20} \mathrm{H}_{10} \mathrm{Cl}_{4} \mathrm{~N}_{4} \mathrm{O}_{2}[\mathrm{M}+\mathrm{Na}]^{+}$500.9450, found 500.9452 .

$\mathrm{N}$-(4-bromophenyl)-2-chloro-5-(5-(3,6-dichloropyridin-2-yl)-1,2,4-oxadiazol-3-yl)benzamide $\mathbf{7 n}$. Yellow solid, yield 69\%, m.p. $242-245{ }^{\circ} \mathrm{C},{ }^{1} \mathrm{H}-\mathrm{NMR}\left(500 \mathrm{MHz}\right.$, DMSO- $\left.d_{6}\right) \delta 10.86(\mathrm{~s}, 1 \mathrm{H}), 8.35$ (d, $J=9.0 \mathrm{~Hz}$, $1 \mathrm{H}), 8.28-8.19(\mathrm{~m}, 2 \mathrm{H}), 7.92(\mathrm{~d}, J=9.0 \mathrm{~Hz}, 1 \mathrm{H}), 7.85(\mathrm{~d}, J=8.5 \mathrm{~Hz}, 1 \mathrm{H}), 7.71(\mathrm{~d}, J=9.0 \mathrm{~Hz}, 2 \mathrm{H}), 7.57$ $(\mathrm{d}, J=8.5 \mathrm{~Hz}, 2 \mathrm{H}) ;{ }^{13} \mathrm{C}-\mathrm{NMR}\left(126 \mathrm{MHz}\right.$, DMSO- $\left.d_{6}\right) \delta 171.72,167.22,163.96,148.59,143.29,139.65$, $138.03,137.52,133.62,131.68,131.24,131.10,129.64,129.24,127.35,124.78,121.67$; HRMS calcd. for $\mathrm{C}_{20} \mathrm{H}_{10} \mathrm{BrCl}_{3} \mathrm{~N}_{4} \mathrm{O}_{2}[\mathrm{M}+\mathrm{Na}]^{+}$544.8945, found 544.8942 .

\subsection{Biological Activity Test}

The insecticidal activity was tested according to [28]. The results of the activity test are shown in Tables 2 and 3. The fungicidal activity of all the synthetic compounds were tested in vitro against eight fungi using a mycelia growth inhibition method according to references [29,30]. Alternaria solani (AS), FusaHum graminearum (FG), Cercospora arachidicola (CA), Phytophthora capsica (PC), Sclerotinia sclerotiorum (SS), Botrytis cinereal (BC), Thanatephorus cucumeris (TC), Fusarium oxysporum (FO) were provided by the National Pesticide Engineering Research Centre, Nankai University. The results of the activity test are shown in Table 4.

\section{Conclusions}

Novel benzamides substituted with pyridine-linked 1,2,4-oxadiazole were designed by bioisosterism, and synthesized easily via esterification, cyanation, cyclization and aminolysis reactions. Through using $\mathrm{CuCN}$ as the cyanidation reagent, L-proline as the catalyst, and increasing the 
temperature gradually in the cyanation reaction, we got the best yield $(79 \%)$ and reduced the risk of this experiment. The structures of the target compounds were confirmed by ${ }^{1} \mathrm{H}-\mathrm{NMR},{ }^{13} \mathrm{C}-\mathrm{NMR}$ and HRMS. The preliminary bioassay results showed that most compounds had good larvicidal activity against mosquito larvae at $10 \mathrm{mg} / \mathrm{L}$, especially compound 7 a with excellent larvicidal activity $(100 \%)$; even at $1 \mathrm{mg} / \mathrm{L}$, the larvicidal actiity was still $40 \%$; at $50 \mathrm{mg} / \mathrm{L}$, all the target compounds showed good fungicidal activity against the eight tested fungi. Compound $7 \mathrm{~h}$ exhibited good inhibitory activity (90.5\%) against Botrytis cinereal, which was better than fluxapyroxad (63.6\%). In addition, it had moderate inhibitory activities against Alternaria solani (50.0\%), Sclerotinia sclerotiorum (80.8\%) and Thanatephorus cucumeris ( $84.8 \%$ ). Therefore, these compounds could potentially be the lead compounds for further optimisation.

Supplementary Materials: The supplementary materials are available online.

Author Contributions: S.Y., X.-Y.T., T.-Y.M., L.D., C.-L.R., J.-C.M. carried out experimental work, S.Y. prepared the manuscript, C.-X.T. designed the material and supervised the project. X.-H.L. and C.-X.T. revised the paper. All authors have read and agreed to the published version of the manuscript.

Funding: This research was funded by the National Key R\&D Program, grant number 2017 YFD0200507.

Acknowledgments: This work was supported financially by the National Key R\&D Program (No. 2017YFD0200507).

Conflicts of Interest: The authors declare no conflict of interest.

\section{References}

1. Liu, X.H.; Yu, W.; Min, L.J.; Wedge, D.E.; Tan, C.X.; Weng, J.Q.; Wu, H.K.; Cantrell, C.L.; Bajsa-Hischel, J.; Hua, X.W.; et al. Synthesis and pesticidal activities of new quinoxalines. J. Agric. Food Chem. 2020. [CrossRef] [PubMed]

2. Alnufaie, R.; Alsup, N.; Whitt, J.; Chambers, A.S.; Gilmore, D.; Alam, A.M. Synthesis and antimicrobial studies of coumarin-substituted pyrazole derivatives as potent anti-staphylococcus aureus agents. Molecules 2020, 25, 2758. [CrossRef] [PubMed]

3. Mu, J.X.; Shi, Y.X.; Yang, M.Y.; Sun, Z.H.; Liu, X.H.; Li, B.J.; Sun, N.B. Design, Synthesis, DFT Study and Antifungal Activity of Pyrazolecarboxamide Derivatives. Molecules 2016, 21, 68. [CrossRef] [PubMed]

4. Liu, X.H.; Qiao, L.; Zhai, Z.W.; Cai, P.P.; Cantrell, C.L.; Tan, C.X.; Weng, J.Q.; Han, L.; Wu, H.K. Novel 4-Pyrazole Carboxamide Derivatives Containing Flexible Chain Motif: Design, Synthesis and Antifungal Activity. Pest Manag. Sci. 2019, 75, 2892-2900. [CrossRef] [PubMed]

5. Wang, L.; Dai, F.Y.; Zhu, J.; Dong, K.K.; Wang, Y.L.; Chen, T. Synthesis and antibacterial activities of pleuromutilin derivatives with thiazole-5-carboxamide and thioether moiety. J. Chem. Res. 2011, 35, 313-316. [CrossRef]

6. Fu, Q.; Cai, P.P.; Cheng, L.; Zhong, L.K.; Tan, C.X.; Shen, Z.H.; Han, L.; Liu, X.H. Synthesis and herbicidal activity of novel pyrazole aromatic ketone analogs as HPPD inhibitor. Pest Manag. Sci. 2020, 76, 868-879. [CrossRef]

7. King, W.F.; Wheeler, R.E. Substituted Oxadiazoles and Their Use as Corn Root Worm Insecticides. U.S. Patent US4237121A, 2 December 1981.

8. Haugwitz, R.D.; Martinez, A.J.; Venslavsky, J.; Angel, R.G.; Maurer, B.V.; Jacobs, G.A.; Narayanan, V.L.; Cruthers, L.R.; Szanto, J. Synyhesis and anthelmintic acyivities of novel isothiocyanatophenyl-1,2,4-oxadiazoles. J. Med. Chem. 1985, 28, 1234-1241. [CrossRef]

9. Liu, Q.; Zhu, R.; Gao, S.; Ma, S.H.; Tang, H.J.; Diao, Y.M.; Wang, H.L.; Zhu, H.J. Structure-based bioisosterism design, synthesis, insecticidal activity and structure-activity relationship (SAR) of anthranilic diamide analogues containing 1,2,4-oxadiazole rings. Pest Manag. Sci. 2017, 73, 917-924. [CrossRef]

10. Terteryan-Seiser, V.; Grammenos, W.; Wiebe, C.; Kretschmer, M.; Craig, I.R.; Escribano, C.A.; Marcus, F.; Tobias, M.; Palomar, M.A.Q.; Grote, T.; et al. Substituted Oxadiazoles for Combating Phytopathogenic Fungi. WO Patent WO2017178245A1, 19 October 2017.

11. Iwata, J.; Nakamura, Y.; Hayashi, T.; Watanabe, S.; Sano, H. Oxadiazole Compound and Fungicide for Agricultural and Horticultural Use. WO Patent WO2019022061A1, 31 January 2019. 
12. Ryu, E.K.; Chung, K.H.; Lee, W.H.; Kim, J.N.; Hong, K.S. Herbicidal Quinolinyloxadiazoles. WO Patent WO9404530A1, 3 March 1994.

13. Nosalova, G.; Strapkova, A.; Korpas, J. Studies of the antitussive effect of prenoxdiazine of experimentally induced cough. Bratisl. Lek. Listy 1982, 78, 47-54.

14. Sakai, K.; Mizusawa, H.; Araki, H.; Higuchi, M.; Yoshikawa, Y.; Tomomatsu, E.; Okajima, Y.; Furukawa, T.; Sakanashi, M.; Atobe, Y. Effects of 5[[2-(diethylamino)-ethyl]amino]-3-phenyl-1, 2, 4-oxadiazole dihydrochloride (DEPO) on cardiovascular system. Oyo Yakuri 1979, 18, 667-672.

15. Zhao, W.; Shen, Z.H.; Xing, J.H.; Yang, G.; Xu, T.M.; Peng, W.L.; Liu, X.H. Synthesis characterization nematocidal activity and docking study of novel pyrazole-4-carboxamide derivatives. Chin. J. Struct. Chem. 2017, 36, 423-428.

16. Hua, X.W.; Liu, W.R.; Su, Y.Y.; Liu, X.H.; Liu, J.B.; Liu, N.N.; Wang, G.Q.; Jiao, X.Q.; Fan, X.Y.; Xue, C.M.; et al. Studies on the Novel Pyridine Sulfide Containing SDH Based Heterocyclic Amide Fungicide. Pest Manag. Sci. 2020, 76, 2368-2378. [CrossRef] [PubMed]

17. Zhao, W.; Shen, Z.H.; Xing, J.H.; Xu, T.M.; Peng, W.L.; Liu, X.H. Synthesis and nematocidal activity of novel pyrazole carboxamide derivatives against meloidogyne incognita. Lett. Drug Des. Discov. 2017, 14, 323-329. [CrossRef]

18. Liu, X.H.; Zhao, W.; Shen, Z.H.; Xing, J.H.; Xu, T.M.; Peng, W.L. Synthesis, nematocidal activity and SAR study of novel difluoromethylpyrazole carboxamide derivatives containing flexible alkyl chain moieties. Eur. J. Med. Chem. 2017, 125, 881-889. [CrossRef] [PubMed]

19. Liu, X.H.; Wang, Q.; Sun, Z.H.; Wedge, D.E.; Becnel, J.J.; Estep, A.S.; Tan, C.X.; Weng, J.Q. Synthesis and insecticidal activity of novel pyrimidine derivatives containing urea pharmacophore against Aedes aegypti. Pest Manag. Sci. 2017, 73, 953-959. [CrossRef]

20. Vishnoi, S.; Agrawal, V.; Kasana, V.K. Synthesis and structure-Activity relationships of substituted cinnamic acids and amide analogues: A new class of herbicides. J. Agric. Food Chem. 2009, 57, 3261-3265. [CrossRef]

21. Ji, W.J.; Xu, T.M.; Zheng, Z.W.; Zhu, B.C.; Li, J.; Hu, W.Q.; Kong, X.L. Synthesis and fungicidal activity of 1-(3-chloropyridin-2-yl)-5-difluorometyl-1H-pyrazole-4-carboxamide derivtives. Chin. J. Pestic. Sci. 2013, 15, 393-397.

22. Bou-Hamdan, F.; Stierli, D.; Jeanmart, S.A.M.; Godfrey, C.R.A.; Hoffman, T.J.; Beaudegnies, R.; Pouliot, M. Oxadiazole Derivatives for Use as Pesticides and Fungicides and Their Preparation. WO Patent WO2017174158A1, 12 October 2017.

23. Hoffman, T.J.; Stierli, D.; Beaudegnies, R.; Pouliot, M.; Pitterna, T. Fungicidal Oxadiazole Derivatives. WO Patent WO2018065414A1, 12 April 2018.

24. Schweizer, E.; Hoffmann-Roeder, A.; Olsen, J.A.; Seiler, P.; Obst-Sander, U.; Wagner, B.; Kansy, M.; Banner, D.W.; Diederich, F. Multipolar interacetions in the D pocket of thrombin:large differences between tricyclic imide and lactam inhibitors. Org. Biomol. Chem. 2006, 4, 2364-2375. [CrossRef]

25. Hamann, L.G.; Manfredi, M.C.; Sun, C.Q.; Krystek, S.R.; Huang, Y.T.; Bi, Y.Z.; Augeri, D.J.; Wang, T.; Zou, Y.; Betebenner, D.A.; et al. Tandem optimization of target activity and elimination of mutagenic potential in a potent series of $\mathrm{N}$-aryl bicyclic hydantoin-based selective androgen receptor modulators. Bioorg. Med. Chem. Lett. 2007, 17, 1860-1864. [CrossRef]

26. Esvan, Y.J.; Zeinyeh, W.; Boibessot, T.; Nauton, L.; Thery, V.; Knapp, S.; Chaikuad, A.; Loaec, N.; Meijer, L.; Anizon, F.; et al. Discovery of pyrido [3,4-g]quinazoline derivatives as CMGC family protein kinase inhibitors: Design, synthesis, inhibitory potency and X-ray co-crystal structure. Eur. J. Med. Chem. 2016, 118, 170-177. [CrossRef]

27. Patrick, D.A.; Bakunov, S.A.; Bakunova, S.M.; Kumar, E.V.K.S.; Lombardy, R.J.; Jones, S.K.; Bridges, A.S.; Zhirnov, O.; Hall, J.E.; Wenzler, T.; et al. Med. Chem. 2007, 50, 2468-2485.

28. Zhang, Y.; Zhu, H.W.; Shang, J.F.; Wang, B.L.; Li, Z.M. Synthesis and Biological Activities of Novel 3-(((3-Bromo1-(3-chloropyridin-2-yl)-1H-pyrazol-5-yl)methylene)amino)-substituted-benzo [d] [1,2,3] triazin-4(3H)-ones. Chin. J. Org. Chem. 2019, 39, 861-866. [CrossRef]

29. Zhai, Z.W.; Shi, Y.X.; Yang, M.Y.; Zhao, W.; Sun, Z.H.; Weng, J.Q.; Tan, C.X.; Liu, X.H.; Li, B.J.; Zhang, Y.G. Microwave Assisted Synthesis and Antifungal Activity of Some Novel Thioethers Containing 1,2,4-triazolo [4,3-a] pyridine. Moiety. Lett. Drug Des. Discov. 2016, 13, 521-525. [CrossRef] 
30. Chen, W.T.; Wang, Q.; Min, L.J.; Wu, H.K.; Weng, J.Q.; Tan, C.X.; Zhang, Y.G.; Hu, B.Z.; Liu, X.H. Synthesis, Crystal Structure, Fungicidal Activity, Molecular Docking, and Density Functional Theory Study of 2-Chloro-N-(p-tolylcarbamoyl) nicotinamide. Indian J. Heterocycl. Chem. 2019, 29, 429-435.

Sample Availability: Samples of the compounds are available from the authors.

(C) 2020 by the authors. Licensee MDPI, Basel, Switzerland. This article is an open access article distributed under the terms and conditions of the Creative Commons Attribution (CC BY) license (http://creativecommons.org/licenses/by/4.0/). 